\title{
AIP
}

\section{Collective thermodiffusion of colloidal suspensions}

Daniel Lüsebrink and Marisol Ripoll

Citation: J. Chem. Phys. 137, 194904 (2012); doi: 10.1063/1.4767398

View online: http://dx.doi.org/10.1063/1.4767398

View Table of Contents: http://jcp.aip.org/resource/1/JCPSA6/v137/i19

Published by the American Institute of Physics.

\section{Additional information on J. Chem. Phys.}

Journal Homepage: http://jcp.aip.org/

Journal Information: http://jcp.aip.org/about/about_the_journal

Top downloads: http://jcp.aip.org/features/most_downloaded

Information for Authors: http://jcp.aip.org/authors

\section{ADVERTISEMENT}

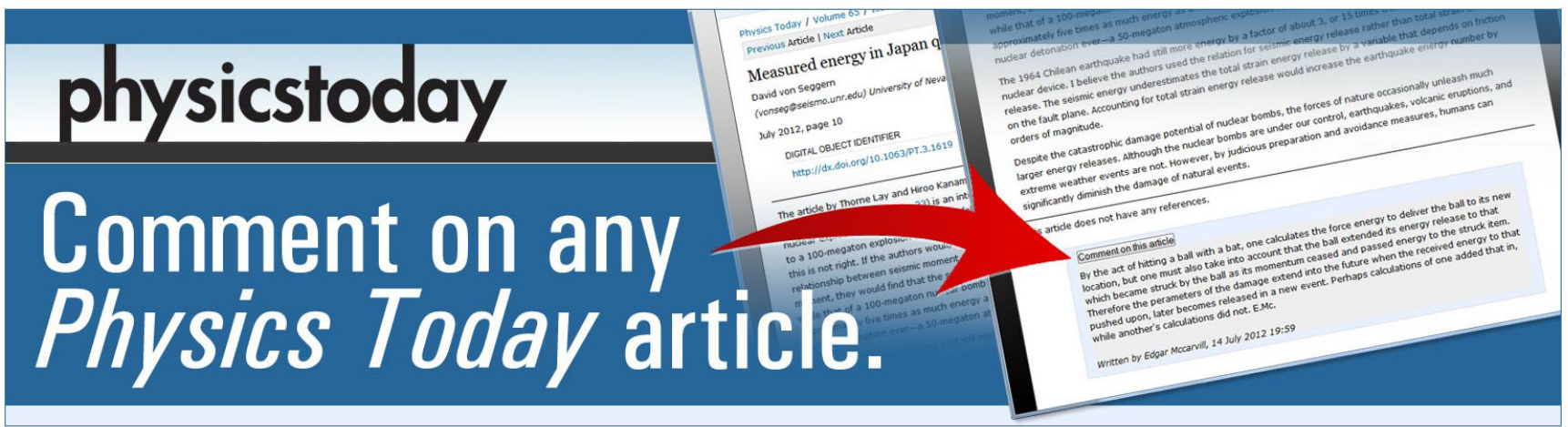




\title{
Collective thermodiffusion of colloidal suspensions
}

\author{
Daniel Lüsebrink and Marisol Ripolla) \\ Theoretical Soft-Matter and Biophysics, Institute of Complex Systems, Forschungszentrum Jülich, \\ 52425 Jülich, Germany
}

(Received 1 August 2012; accepted 25 October 2012; published online 21 November 2012)

\begin{abstract}
The thermophoretic behavior of concentrated colloidal suspensions can be understood as the sum of single particle and collective effects. Here, we present a simulation model to investigate the particularities of the collective thermodiffusive effects in concentrated uncharged solutions, where the influence of different colloid-colloid interactions is analyzed. The concentration dependence found in our simulations qualitatively agrees with experimental results. Colloids with repulsive interactions are found to accumulate more effectively than the solvent in the warm areas, such that the corresponding Soret coefficients are negative and decrease with increasing concentration. The accumulation of colloids in the cold regions is facilitated by attraction, such that colloids with attractive interactions have larger values of the Soret coefficient. A thermodynamic argument that explains our results from equilibrium quantities is discussed as well. () 2012 American Institute of Physics. [http://dx.doi.org/10.1063/1.4767398]
\end{abstract}

\section{INTRODUCTION}

The transport of mass as a response to a temperature gradient is known as thermodiffusion or Soret effect. ${ }^{1}$ This phenomenon has found a large number of applications in general and, in particular, when applied to soft matter systems such as colloidal or polymeric dispersions. Examples range from separation techniques ${ }^{2,3}$ to microfluidic applications. ${ }^{4-6}$ Moreover, thermodiffusion might even have facilitated conditions for the origin of life. ${ }^{7,8}$ Most of the existing work on thermodiffusion of colloidal systems is performed in the regime of dilute systems, ${ }^{9-15}$ where the interactions between macromolecules are not important. The scarce theoretical and experimental investigations have mainly focused on strongly interacting systems and have concluded that there is a pronounced concentration dependence of the thermodiffusive behavior. We briefly summarize various aspects of these studies. Experiments with micellar systems ${ }^{16}$ display a linear dependence of the inverse Soret coefficient on concentration, which is reversed with the addition of surfactant. Measurements on polystyrene solutions ${ }^{17}$ show a strong dependence of some thermodiffusive properties as the system approaches the glass transition, while the Soret coefficient decreases slowly with the concentration, insensitive to the glass transition. Concentrated charged colloidal systems have been investigated experimentally and theoretically. ${ }^{18,19}$ In all presented cases, these systems are characterized by a negative Soret coefficient that increases with concentration. Using a microscopic approach at low volume fractions, the contribution of colloidcolloid interactions to the thermal diffusion coefficient has been studied theoretically. ${ }^{20,21}$ The temperature dependence of the potential of mean-force has shown to give rise to sign changes of the Soret coefficient upon changing temperature and/or concentration under appropriate conditions. Recent experiments $^{22}$ have investigated the thermophoretic properties of concentrated colloidal suspensions in which the

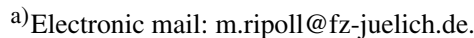

interactions between the colloids were varied from purely repulsive to short ranged attractive. Results in the low concentration regime show a reasonable agreement with theory, although at larger concentrations a significant change of behavior is observed. At low concentrations, the contribution of the colloid-colloid interactions or collective contribution is found to be much less important than the contribution of the colloidsolvent interaction or single particle contribution. However, not much is known about the origin of the behavioral change at larger concentrations. A detailed study of the collective contribution to the thermodiffusion of colloidal systems by means of computer simulations can, therefore, importantly contribute to the deeper understanding of these systems.

In this work, simulations of concentrated systems are performed with different colloid-colloid interaction potentials. The model used here essentially disregards single particle effects becoming an ideal candidate to study the importance of collective interactions in the thermodiffusion phenomena. The paper is organized as follows. After a brief introduction of the main theoretical concepts, we present and reinterpret the experimental results of Ning et al. ${ }^{22}$ to display more clearly the importance of the collective contribution. Then, the simulation model and the procedure to perform the measurements is introduced, and the simulation results are presented afterwards. The influence of the different characteristics of the interaction potentials are analyzed separately, such as the effect of the steepness of the repulsive potential, the range, or the strength of the attractive interaction. Finally, we present a simple thermodynamic argument that allows us to express the Soret coefficient of the dispersion in terms of the thermal expansion of its components, which explains some of our results very satisfactorily.

\section{THEORY}

The thermodiffusion phenomena of a two component mixture can be characterized phenomenologically by the 
quantification of the particle flux $J$ of one of the components in the direction of a temperature gradient $\nabla T,{ }^{23}$

$$
J=-\tilde{n} D_{m} \nabla x-\tilde{n} D_{T} x(1-x) \nabla T,
$$

where $\widetilde{n}=n+n^{\prime}$ is the averaged total number density, with $n$ and $n^{\prime}$ are the density of the two components, $x=n / \tilde{n}$ is the molar fraction of component one, $D_{m}$ is the mutual diffusion coefficient, and $D_{T}$ is the thermal diffusion coefficient. The so-called Soret coefficient is defined as the ratio of the two diffusion coefficients

$$
S_{T} \equiv \frac{D_{T}}{D_{m}}
$$

and indicates how strongly the two components separate. In the stationary state, the particle flux vanishes, and the Soret coefficient can be obtained from the molar fraction and temperature distribution

$$
S_{T}=-\frac{1}{x(1-x)} \frac{\nabla x}{\nabla T}
$$

Note that by convention, a positive $S_{T}$ indicates that the relative accumulation of component one is higher in the cold side, while a negative $S_{T}$ will display a reverse behavior. Equation (3) constitutes the standard method to quantify the thermodiffusion phenomena in concentrated mixtures. Recently ${ }^{24}$ an alternative expression of the Soret coefficient has been proposed in terms of the difference of mechanical driving forces exerted on the particles. These forces are induced by the presence of temperature inhomogeneities on the constituent particles, namely, the thermophoretic forces $\mathbf{f}$ and $\mathbf{f}^{\prime}$,

$$
S_{T}=\left(\mathbf{f}^{\prime}-\mathbf{f}\right) \frac{1}{k_{B} T \nabla T} .
$$

In the case of a large size separation between the components, as is the case of a colloidal suspension, the force on the small component $\mathbf{f}^{\prime}$ can be neglected.

When investigating the thermodiffusion properties of a colloidal suspension with the volume fraction $\phi$, the behavior can be understood to include two main contributions. The single particle contribution is determined by the particular characteristics of the local interactions of the colloid with the surrounding solvent and can be characterized in the limit of diluted solutions. The collective contribution appears when the number of colloids in the solution increases and will be determined by both the interactions among colloids and eventually also by the interactions of the colloid with the surrounding solvent. The single particle contribution of the Soret coefficient has shown ${ }^{13,25-27}$ a pronounced dependence on the average temperature, particle size, or nature of the surface interactions.

The collective interactions have been studied theoretically by Dhont. ${ }^{20,21}$ This theory predicts that colloids with hard sphere interactions in an incompressible solvent, display at low concentrations, an approximately linear decay of the Soret coefficient with the volume fraction $\phi$ that can be de- scribed by the expression

$$
\begin{aligned}
S_{T}^{\text {low }}(\phi) & =\frac{1}{T} \frac{1-0.35 \phi}{(1-\phi)(1+1.45 \phi)}+\frac{D_{T, \text { sing }}}{D_{0}(1+1.45 \phi)}, \\
& \simeq \frac{1}{T}(1-0.80 \phi)+S_{T, \text { sing }}(1-1.45 \phi),
\end{aligned}
$$

where $D_{0}$ is the translational diffusion coefficient in the limit infinite dilution. Here, $D_{T, \text { sing }}$ and $S_{T, \text { sing }}$ refer to the single particle contribution of the thermal diffusion and the Soret coefficients, respectively. The difference between $S_{T, \text { sing }}$ and $S_{T}^{\text {low }}(\phi=0)$ corresponds to the ideal gas contribution. ${ }^{28} \mathrm{Com}-$ parison of this theory with experimental results in the low concentration regime is discussed in Sec. III. For larger volume fractions, there is to our best knowledge no available theory for uncharged colloidal systems.

In the theoretical approach of Dhont, ${ }^{20,21}$ the single particle and the collective contributions to the thermal diffusion coefficient are assumed to be independent of each other, this is $D_{T}=D_{T, \text { sing }}+D_{T \text {, coll }}$, and similarly for the mutual diffusion coefficient. Considering the definition of the Soret coefficient in Eq. (2), it can be easily inferred that the single particle contribution can be defined as $S_{T, s i n g}$ $=D_{T, \text { sing }} / D_{0}$. This is not the case for the collective contribution, $S_{T, \text { coll }}$, which will be, in general, dependent on both $D_{T, \text { sing }}$ and $D_{T, \text { coll }}$. Alternatively, it could be assumed that $S_{T, \text { sing }}$ and $S_{T, \text { coll }}$ are independent of each other, which would imply that the two components of $D_{T}$ would not be independent then. This assumption can be justified considering that the related thermophoretic forces in Eq. (4) in a concentrated suspension could be considered as the sum of two independent contributions. Simulations and experiments considering different interactions between colloids and solvent as well as different colloid-colloid interactions at varying concentration can help us to understand this point better.

\section{EXPERIMENTAL RESULTS}

The thermal diffusion behavior of coated colloids in toluene as a function of the volume fraction has been experimentally investigated by Ning et al. ${ }^{22}$ The coating consists of octadecyl alcohol, which are chains with a length of 18 carbon groups. They can be thought of as a thin brush covering the colloid core. The core interactions are essentially attractive (van der Waals interactions) and, the coating of the colloids is typically employed to stabilize the suspension. The brush hair has a length roughly $1 \%$ of the colloid size, and a temperature dependent behavior that can change the colloid properties. At low temperatures, the attached brushes are in a collapsed state, such that the colloid-colloid interactions are attractive at short distances. ${ }^{29,30}$ At high temperatures, the brushes tend to be extended, and therefore the colloid-colloid interactions are close to a hard spherelike behavior.

Experiments at different temperatures and volume fractions of colloids are performed by Ning et al. ${ }^{22}$ The experimental results of the Soret coefficient are summarized in Fig. 1(a). The range of concentrations goes from the very dilute solutions with about $1 \%$ volume fraction of colloids up to $30 \%$ volume fraction. In the range below $10 \%$ the magnitude 

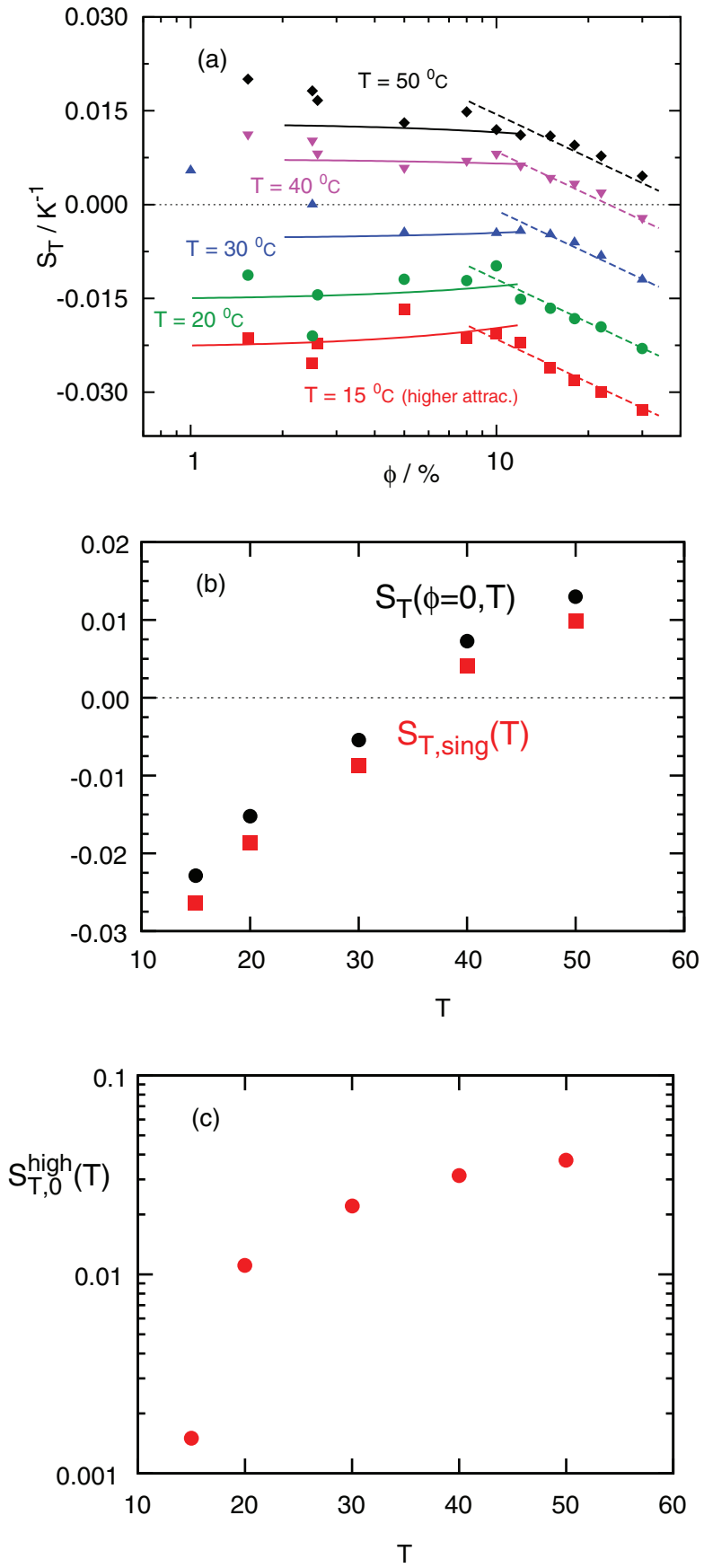

FIG. 1. (a) Soret coefficient for octadecyl coated silica particles in toluene as a function of the volume fraction $\phi$ for different values of the average temperature. Solid lines are a fit to Eq. (5) for low $\phi$, and dashed lines a fit to Eq. (7) for high $\phi$. (b) Values of the Soret coefficient at the limit of zero volume fraction, and the single particle contribution as a function of the temperature, obtained from the fit of the data to Eq. (5). (c) Temperature dependence contribution of $S_{T}$ at high $\phi$ values are obtained from a fit of the data to Eq. (7). (a) Reprinted with permission from J. Chem. Phys. 125, 204911 (2006). Copyright 2006 American Institute of Physics.

of the Soret coefficient decreases slightly in all cases. In this regime, the experimental data are compared to the theoretical results in Eq. (5) shown as solid lines. This comparison allows them to determine the values of the single particle contribution $S_{T, s i n g}$ in Eq. (6). The extrapolation to zero concentration $S_{T}(\phi=0)$ can also be straightforwardly obtained from Eq. (6). Both values are presented in Fig. 1(b), where a strong increase

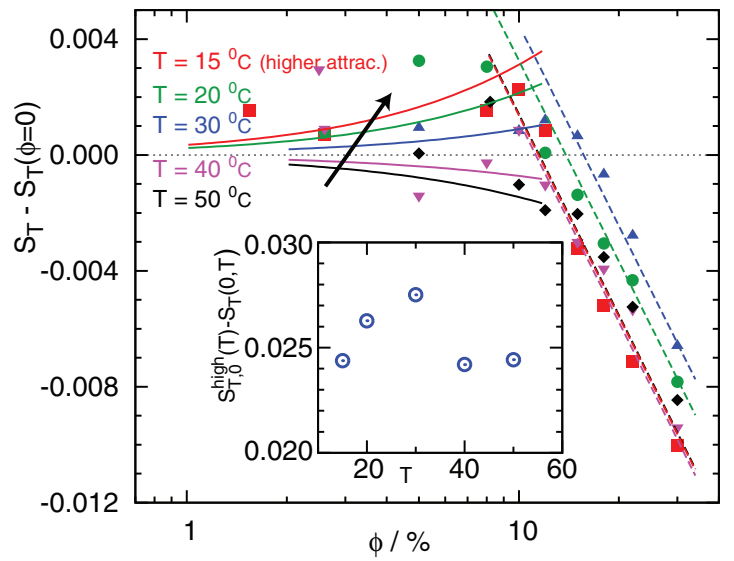

FIG. 2. Displaced Soret coefficient as a function $\phi$, corresponding to the collective contribution. Lines correspond to those in Fig. 1. The arrow indicates increasing colloid-colloid attraction. (Inset) Difference between the "zero concentration extrapolated Soret coefficients" at low concentration in Eq. (6) and high concentration in Eq. (7).

of $S_{T}$ with temperature can be observed. Similar behavior is experimentally observed for colloids in aqueous solutions ${ }^{27}$ in the limit of very low concentrations.

For larger volume fractions, the dependence of $S_{T}$ with concentration changes significantly, and the theory of Dhont is not longer valid. In Fig. 1(a), it can be seen that for volume fractions larger than $10 \% S_{T}$ becomes a decreasing function in all cases, eventually even displaying a sign change. Furthermore, the functional form of this decay can be empirically described by a universal logarithmic decay ${ }^{31}$

$$
S_{T}^{h i g h}=-0.01 \ln \phi+S_{T, 0}^{h i g h} .
$$

The extrapolated value at zero concentration $S_{T, 0}^{\text {high }}$ accounts for a constant displacement including all the temperature dependence of $S_{T}^{\text {high }}$. This extrapolated value is noticeably different from the limit at vanishing concentration of $S_{T}^{l o w}$ as displayed in Fig. 1(c).

As shown in Fig. 1, single particle effects have a very strong contribution to the Soret coefficient for all volume fractions. It is interesting though to find a representation in which these effects are disregarded such that the behavior of the purely collective effects can be investigated. For this purpose, Fig. 2 compares the different sets of data displacing them all to the same vanishing value at limiting zero concentration. At low volume fractions, the collective contribution to the Soret coefficient in Fig. 2 decreases with increasing temperature. This trend follows from the analytical curves in Eq. (5) as a consequence of the different $S_{T, s i n g}$ contributions. Within this representation, the relative error of experimental data is magnified in the low concentration regime as can be seen in Fig. 2. On the other hand, increasing the temperature of the system employed in Ref. 22 affects also the colloid-colloid interactions that vary from hard-sphere type of repulsion at high temperature $\left(50^{\circ} \mathrm{C}\right)$, to strong short-ranged attractive interactions at low temperatures $\left(15^{\circ} \mathrm{C}\right)$. From the data at low concentrations in Fig. 2 it can then be understood that increasing the strength of the attraction increases the value of the Soret coefficient, or similarly, that with increasing attraction the colloids accumulate more effectively in the cold areas. Moreover, 
the collective contribution to the Soret coefficient is typically negative for purely repulsive colloids and positive for colloids with a short-range attraction.

The logarithmic decay described in Eq. (7) in the regime of large concentrations is independent of the temperature. Nevertheless, the relative displacement of these curves with respect to each other shows to be non-monotonic with temperature. This can be seen directly in the data in Fig. 2, and in the differences of the Soret coefficients extrapolated to zero concentration for low concentrations in Eq. (6) and high concentrations in Eq. (7), as displayed in the inset of Fig. 2. This non-monotonic behavior will be most likely overruled by the experimental error when determining the contribution to the Soret coefficient in the limit of zero concentration, since these values were not the main goal of the experiments in Ref. 22. More precise experimental data, and/or simulation results, can contribute to clarify whether the behavior at high concentrations depends on the interaction, and in that case if it has the same, or opposite trend as in the low concentration regime. This is, whether increasing attractive interactions facilitate the effective accumulation of colloids in the cold areas.

\section{SIMULATION MODEL}

Existing simulations of colloids in temperature gradients $^{32-34}$ are performed with molecular dynamics (MD) of both the colloidal and solvent particles. The typical experimental sizes of colloids and solvent particles can be separated by several orders of magnitude. This largely hinders simulations that explicitly account for the solvent particles such as MD or Monte Carlo. Alternatively, several methods to coarse grain the solvent dynamics have been developed in the last decades. Outstanding examples are lattice Boltzmann (LB) ${ }^{35}$ or dissipative particle dynamics (DPD). ${ }^{36}$ Both these methods are isothermal in their most extended implementations, although corresponding modifications to these models have been proposed in order to be able to sustain temperature inhomogeneities both for $\mathrm{LB}^{37,38}$ and DPD. ${ }^{39-41}$

In the present work, we employ a relatively new coarse-grained method known as multiparticle collision dynamics (MPC) ${ }^{42}$ or stochastic rotation dynamics. ${ }^{43,44}$ This technique has shown to include properly hydrodynamic interactions, ${ }^{45,46}$ and furthermore to be able to sustain temperature gradients. ${ }^{47}$ Colloidal dynamics are simulated by MD, where the interactions between colloids is considered by explicit potentials. Apart from the explicit and efficient implementation of the non-isothermal solvent, the employed MPC method offers us the possibility of strongly tune the solvent colloid interactions.

\section{A. MPC solvent}

The mesoscopic MPC fluid consists in $N$ point particles of constant mass $m_{i}$ (with $i$ the particle index) in a box of volume $V$. The particle state is described by its position $\mathbf{r}_{i}$ and velocity $\mathbf{v}_{i}$ which vary continuously in phase space. Two alternating steps determine the system dynamics. In the streaming step, particles evolve ballistically during a certain collision time $h, \mathbf{r}_{i}(t+h)=\mathbf{r}_{i}(t+h)+h \mathbf{v}_{i}(t)$. In the collision step, particles are sorted into cubic collision boxes. All particles $j$ within the collision box of particle $i$ at a certain time step, interact with each other through the center-of-mass velocity of the collision box

$$
\mathbf{v}_{c m, i}(t)=\frac{\sum_{j}^{i, t} m_{j} \mathbf{v}_{j}(t)}{\sum_{j}^{i, t} m_{j}} .
$$

The actual collision considers a rotation of the relative velocity to the center-of-mass velocity by an angle $\alpha$, around a random orientation,

$$
\mathbf{v}_{i}(t+h)=\mathbf{v}_{c m, i}(t)+\mathcal{R}(\alpha)\left[\mathbf{v}_{i}(t)-\mathbf{v}_{c m, i}(t)\right],
$$

with $\mathcal{R}(\alpha)$ the stochastic rotation matrix. This simple collision rule imposes conservation of mass, linear momentum, and kinetic energy at the collision box level. This ensures the presence of hydrodynamic interactions, together with the sustainability of temperature gradients, and thermal fluctuations. In order to preserve Galilean invariance, and to enhance collisional transport, random shift of the collision box grid is additionally considered. ${ }^{43}$ The reference units are chosen to be the particle mass $m$, the collision box size $a$, and a reference system temperature $T$, that are set to one. This corresponds to measure length as $\hat{x}=x / a$ and time as $\hat{t}=t \sqrt{k_{B} T / m a^{2}}$, with $k_{B}$ the Boltzmann constant. The transport properties of the MPC fluid have been very successfully related to the MPC parameters by means of kinetic theory calculations. ${ }^{48-50}$ These MPC parameters are the collision time $h$, the rotation angle $\alpha$, and the particle density $\rho=N / V$. The MPC solvent is characterized by the ideal gas equation of state due to the absence of an explicit interaction potential among its constituent particles. Nevertheless, the range of parameters with large values of $\alpha$ and small values of $h$ has shown to display a liquidlike behavior characterized by large values of the Schmidt number $S c=v / D$ with $v$ the kinematic viscosity and $D$ the diffusion coefficient. ${ }^{46,51}$ In this work, we use a unique set of parameters to describe the MPC solvent, these are $h=0.1, \alpha=120^{\circ}$, and $\rho=5$, which in three dimensions corresponds to $S c=9.6$. The averaged temperature has been set to $T_{0}=2$ in all simulations, unless stated otherwise.

\section{B. Colloid-solvent interactions}

In this work, we employ a simplified model for simulating the interaction of the colloids with the surrounding solvent. The interaction of solvent particles and colloids occurs in the collision step, in which the colloids are considered as point particles of different mass than the fluid particles in the calculation of the collision box center-of-mass velocity in Eq. (8). This is the MPC coupling. The fact that colloidsolvent interactions do not have any exclude volume potential implies that the solvent particles can penetrate the volume of the colloids since they perceive them only as heavy point particles. A consequence is that local effects are mostly not taken into account.

In a recent study, performed in parallel to the present one, colloids interacting with MPC solvent and non-penetrating 
solvent-colloid interactions ${ }^{52,53}$ have been investigated in the limit of an infinitely dilute solution. In this case, the single particle contribution to thermodiffusion shows an important dependence on the solvent-colloid interactions which can even change direction. Simulations with MD solvent and colloids have also investigated different features of their behavior in the presence of temperature gradients, ${ }^{32-34}$ but have so far been only employed in the dilute regime.

In the present work, we restrict ourselves to the MPC coupling for colloid solvent interactions. In spite of its drawbacks, this coupling has shown to capture the dynamics of solute particles ${ }^{51}$ and has been used in similar fashion to correctly describe some aspects of the dynamics of concentrated colloidal solutions. ${ }^{51,54}$ One of the most successful characteristics of the method and this coupling is that hydrodynamic interactions are properly taken into account. This has been verified in dilute polymer solutions ${ }^{46,55}$ simulated with this coupling, and it has been frequently employed in systems such as rodlike colloids, ${ }^{56-59}$ star polymers, ${ }^{60-62}$ or self-propelled structures. ${ }^{63-65}$

More interestingly, the MPC coupling provides a very useful opportunity to investigate the collective contribution to the thermodiffusion effect in colloidal suspensions, since the single particle contribution is strongly suppressed, as will be precisely shown in Sec. V A 1. Additionally, this coupling is computationally very efficient, given the fact that explicit solvent-colloid forces do not need to be calculated.

\section{Colloid-colloid interactions}

A pair interaction potential between colloids is considered by means of MD. This means that although the colloids take part in the MPC collision step, they do not follow the MPC streaming step. ${ }^{45}$ The colloids positions are updated with the MD integration algorithm, velocity-Verlet in our case, where the potential interactions are considered. The MD integration time step $\Delta t$ should be small enough to integrate the corresponding potential, although there is no further dependence on it. Typical values are $h / \Delta t=50,100,200$ depending on the potential.

The colloid-colloid pair potentials are varied to analyze the influence of attractive and repulsive interactions, and the effects of softness or attraction strength. Two functional forms for the potentials are employed in this work. One is the Lennard-Jones (LJ) type potentials ${ }^{66}$

$$
U(r)=4 \epsilon\left[\left(\frac{\sigma}{r}\right)^{2 n}-\left(\frac{\sigma}{r}\right)^{n}\right]+C, \quad r<r_{c}
$$

with $r$ the distance between two colloid centers. The potential depth is by default chosen to be $\epsilon / k_{B} T=1$, with $k_{B} T$ the reference temperature. The colloidal diameter is fixed to $\sigma$ $=1$, and the mass is $m_{c}=5.0$; this is the value that matches the mass of the fluid in one collision box which has shown to be an appropriate value to enhance the effect of hydrodynamic interactions. ${ }^{51}$ The repulsive potential (rLJ) is characterized by $C=\epsilon$, and the attractive (LJ) by $C=0$. The value of the exponent $n$ determines the softness and range of the interaction. The cut-off radius for rLJ is simply $r_{c}=2^{1 / n} \sigma$ and for the attractive LJ interaction is matched at the point where the potential is as small as the standard $\mathrm{LJ} n=6$ potential. In this way, we define $\widetilde{U}=U^{n=6}(r=2.5 \sigma)$, such that $r_{c}=[2 /(1-\sqrt{1+\widetilde{U}})]^{1 / 6} \sigma$.

The second functional form of the potential is intended to more accurately match the experimental interactions in Ref. 22, which are very short ranged. To this potential we refer as sticky potential, $U^{s t k}(r)$, in correspondence to the sticky spheres in experiments. Similar to the Lennard-Jones potential, the sticky potential is composed by two terms, a repulsive core and an attractive tail. The repulsive core is given by

$$
U_{r}^{s t k}\left(r_{i j}\right)=\lambda_{r}\left(\frac{\sigma}{r_{i j}}\right)^{48}
$$

and the attractive part that is chosen to reproduce the functional form of depletion interactions, ${ }^{67-69}$

$$
U_{a}^{s t k}\left(r_{i j}\right)=-\lambda_{a}\left[1-\frac{3}{4}\left(\frac{r_{i j}}{a}\right)+\frac{1}{2}\left(\frac{r_{i j}}{2 a}\right)^{3}\right] .
$$

The coefficients $\lambda_{r}$ and $\lambda_{a}$ determine the relative strength of the two contributions. The colloid diameter is estimated by $\sigma$, and the range of the attractive part of the potential is independently determined by $a$. The total potential is given by

$$
U^{s t k}\left(r_{i j}\right)=\epsilon\left[U_{r}^{s t k}\left(r_{i j}\right)+U_{a}^{s t k}\left(r_{i j}\right)-U_{r}^{s t k}\left(r_{c}\right)\right],
$$

where $\epsilon$ is the strength of the potential, and $r_{c}$ is the cutoff radius. The parameters employed in the simulations in Sec. V are specified in Table I.

In the simulations, the number of colloids $N_{c}$ in the simulation box of size $V$ is varied to account for concentration effects. The volume fraction of colloids is defined as $\phi_{c}=N_{c} \pi \sigma^{3} / 6 \mathrm{~V}$. The effective diameter is not taken into account, although this should not have a big effect, since the estimation of the effective diameter with an expression, such as Barker-Henderson, ${ }^{70}$ has shown to increase the diameter only by $1 \%$ with our choice of parameters. ${ }^{51}$

\section{Temperature gradient establishment}

We consider a three-dimensional system with periodic boundary conditions. In one of the dimensions, the temperature gradient is realized by defining a cold layer with temperature $T_{c}$ at one extreme of the box, and a hot layer with temperature $T_{h}>T_{c}$ at the center of the box. In this way, the simulation box is divided in two half-boxes with increasing temperatures towards the center. The temperature at the cold and hot layers is not directly imposed but a consequence of an energy flux which is imposed with the so-called velocity

TABLE I. Parameters employed for the sticky potentials in Eq. (13).

\begin{tabular}{llcl}
\hline \hline$r_{c} / \sigma$ & $\lambda_{r}$ & $\lambda_{a}$ & \multicolumn{1}{c}{$a / \sigma$} \\
\hline 1.0365 & 4. & 1940. & 0.522 \\
1.1 & 1.3 & 185. & 0.55 \\
1.2 & 1.0 & 43. & 0.6 \\
\hline
\end{tabular}




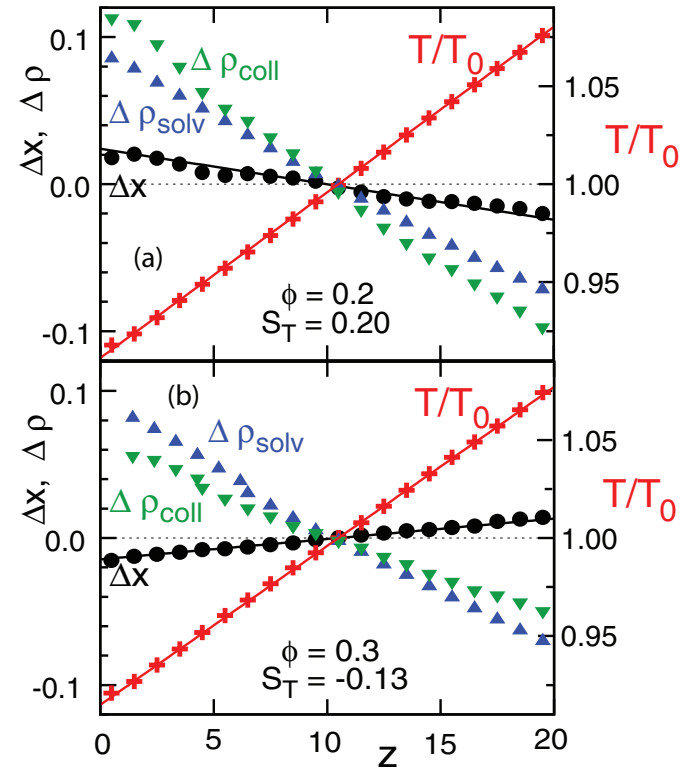

FIG. 3. Profiles for concentrated colloids suspensions interacting with the LJ $n=6$ potential. Crosses indicate the temperature profile with values in the right axis. The left axis quantifies the normalized relative density of colloids (down triangles), solvent particles (up triangles), and the normalized relative molar fraction of colloids (bullets). Lines correspond to the linear fits to determine the gradients of temperature and molar fraction. (a) $\phi_{c}=0.2$ as example of a positive $S_{T}$ with colloids excess on the cold side. (b) $\phi_{c}=0.3$ as example of a negative $S_{T}$.

exchange algorithm. The method was originally introduced in the framework of MD simulations, ${ }^{71,72}$ and consists in identifying the hottest solvent particle of the cold layer and the coldest of the warm layer, and interchange their velocities, as a type of a Maxwell's demon. More details of this, and other possible implementations of the temperature gradient within the MPC fluid can be found in Ref. 47. After a certain time, the system in contact with the thermal baths reaches a steady linear temperature profile

$$
T(z)=T_{c}+\nabla T z
$$

with $\nabla T=\left(T_{h}-T_{c}\right) / L_{z}$ the resulting temperature gradient in the z-direction, and $L_{z}$ the distance between the two baths. The equation of state of a MPC fluid is the one of an ideal gas which determines the density distribution as $\rho(z)=p / k_{B} T(z)$, with $p$ the system pressure. In the limit of small temperature differences, the density profile can be approximated to a linear function decreasing in the direction of the increasing temperature. Typical examples of the temperature and solvent density profiles are shown in Fig. 3. The size of the simulation box is $L_{\perp}$ in the two directions perpendicular to the temperature gradient and $2 L_{z}$ in the gradient direction. In this work, we have fixed $L_{z}=40$ for all potentials. For the purely repulsive interactions we use $L_{\perp}=30$. For the attractive potentials we employ the smaller value $L_{\perp}=10$, in order to reduce computational costs. Simulations are started by arranging the colloidal particles on a grid and letting the system relax to a stationary state. Similar to the experimental values we have varied the volume fraction of colloids up to $\phi_{c}=30 \%$.

\section{E. Determination of the Soret coefficient}

The Soret coefficient is determined through Eq. (3) by measuring the temperature and the density distribution profiles of both colloids and solvent within the simulation box. The profiles are averaged over simulation steps such that local fluctuations in temperature and density are reduced. For further analysis, the first two boundary slabs, where the velocity exchange is performed, are disregarded.

The average density $\rho_{k}$, is the density that component $k$ would have in the absence of a temperature gradient. It is instructive to show in which percentage each component varies its density with respect to the average value. The normalized relative density is then defined as

$$
\Delta \rho_{k}(z)=\frac{\rho_{k}(z)-\rho_{k}}{\rho_{k}}
$$

Figure 3 shows two examples of relative density profiles of the solvent and colloids together with their corresponding temperature profiles. The profiles are only shown for the left side of the bi-periodic simulation box, although the averages consider both boxes. The density profile of the fluid essentially does not change due to the presence of the colloids and it is determined by the ideal gas equation of state. The average colloidal molar fraction $x_{c}=\rho_{c} /\left(\rho_{c}+\rho_{s}\right)$ and its gradient can then be determined from the density values. The resulting normalized relative mass fractions are shown in Fig. 3 for two values of the colloid volume fraction. These values together with the temperature gradient and Eq. (3) allows us to determine the Soret coefficient, as shown in Fig. 3. It is interesting to note that

$$
\Delta \rho_{c}(z)>\Delta \rho_{s}(z) \Leftrightarrow \Delta x_{c}(z)>0 .
$$

This means that if the colloids have a relative density variation larger than the solvent in the cold part of the system, the weight fraction decreases with increasing temperature, or similarly that $S_{T}>0$. This is the case displayed in Fig. 3(a), while the reverse situation can be seen in Fig. 3(b). Therefore, in this system it is not adequate to say that $S_{T}<0$ indicates that the component is thermophilic or that it goes preferably to the warm side, as it would happen, for example, in an incompressible fluid. Both components can accumulate in the cold side, but one can accumulate less effective than the other one in the cold side, which would lead to a negative $S_{T}$.

The values obtained for $S_{T}$ are given in MPC units, as indicated in Sec. IV A. In order to compare these values with experimental ones, the LJ values can be referred to the Argon values, as standardly done with MD simulations. This choice is $\epsilon_{A r}=999 \mathrm{~J} / \mathrm{mol}$, which implies that the relevant factor to convert the units is $\epsilon_{A r} / T R=60 \mathrm{~K}$ with $R=8.314 \mathrm{~J} /(\mathrm{K} \mathrm{mol})$ the ideal gas constant, and $T=2$ the employed average temperature. The typical values obtained in our investigation are between $S_{T} \simeq 0.2$ and $S_{T} \simeq-0.3$ in MPC units which correspond to values between $S_{T} \simeq 0.003 \mathrm{~K}^{-1}$ and $S_{T} \simeq-0.005 \mathrm{~K}^{-1}$. This gives a reasonable quantitative agreement with the experimental data in Fig. 2. 


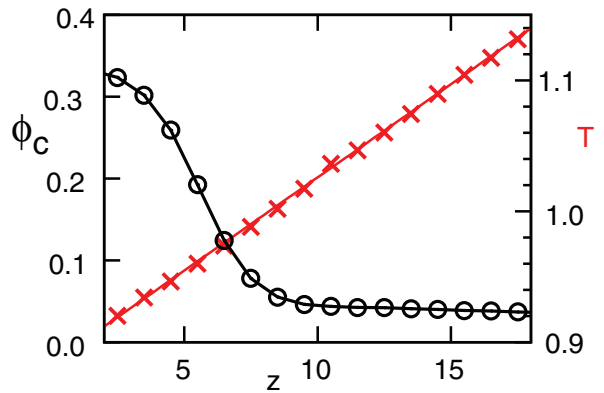

FIG. 4. Temperature gradient induced phase separation of colloids in liquidgas coexistence regime below critical point with $\mathrm{LJ} 12-6, \epsilon=1.0, \phi_{c}=0.1$, and $T_{0}=1.0$. The volume fraction on the cold side of the system is $\phi_{c} \simeq 0.37$ corresponding to a liquid state. On the hot side, $\phi_{c} \simeq 0.03$ corresponding to a gas phase.

\section{F. Condensation effects}

The absolute values of the temperature distribution may drive the cold and hot sides of the system into different points of the phase diagram. The Monte Carlo simulations by Vliegenthart et al. ${ }^{66}$ of Lennard-Jones fluids have shown that the critical point of a $\mathrm{LJ} n=6$ fluid is at $T_{c}=1.316$ and $\phi_{c}=0.165$. where $\phi_{s c} \approx 0.52$ is the simple cubic packing fraction. Below the critical point, the system is in the liquidgas coexistence regime and a phase separation due to the temperature gradient is observed as can be seen in Fig. 4. In this example, where the average temperature is below the critical temperature the volume fraction profile of the colloids is not changing linearly, but drops abruptly indicating a liquid phase of colloids on the cold side and a gas phase on the warm side. In principle it is also possible that the colloids form clusters or droplets, structures that might not be easily observed in density profiles. For simulation parameters below the critical point (i.e., high attraction strengths or low temperatures), we have checked that there are no additional colloid accumulations, besides the one observed in Fig. 4. We assume that this check is sufficient although a more exhaustive analysis would require, for instance, to investigate the pair correlation functions. In any case, for the remaining study of thermodiffusion effects performed in this work, we chose system parameters such that the system remains in a single phase.

\section{SIMULATION RESULTS}

To study collective effects in the thermodiffusion of colloidal suspensions, we perform simulations for various colloid-colloid interactions with varying colloidal volume fractions. Different characteristics of the interactions and their effects on the Soret coefficient are investigated. The purely repulsive or attractive nature of the interaction, the steepness of the repulsion, the range, and the strength of the attractive interaction are the effects we analyze.

\section{A. Limiting cases}

\section{Limit of infinite dilution}

In the limit of very low volume fractions, there are no interactions among colloids, such that the values of $S_{T}$ should
TABLE II. Values of the Soret coefficient for the MPC binary mixture at various concentrations, and for two potential interactions at very low concentrations.

\begin{tabular}{llll}
\hline \hline Interaction & $x_{c}$ & $\phi$ & $S_{T}$ \\
\hline MPC binary mixture & 0.02 & 0.05 & 0.033 \\
“ & 0.04 & 0.1 & 0.019 \\
“ & 0.07 & 0.2 & 0.021 \\
“ & 0.10 & 0.3 & 0.017 \\
$\mathrm{rLJ} n=6$ & & 0.001 & 0.02 \\
$\mathrm{LJ} n=6$ & & 0.001 & 0.01 \\
\hline \hline
\end{tabular}

be independent of the interaction potential. This is the single particle limit in which $S_{T}$ is determined by the colloid-solvent interactions. In order to quantify $S_{T}$ with the MPC coupling in this limit we perform two types of simulations. First, interactions potential among colloids are not taken into account, and second the limit of very low volume fraction is investigated.

In case colloids without interaction potential are considered, the colloids are reduced to be heavy particles, this is MPC particles of larger mass than the solvent particles, such that the system is a binary mixture of MPC particles. Values computed for $S_{T}$ in such MPC binary mixture, and various concentrations are shown in Table II. The concentrations are expressed in terms of the heavy particle molar fraction $x_{c}=N_{c} /\left(N_{c}+N\right)$. For the sake of better comparison, we also refer to the volume fraction of $N_{c}$ colloids of diameter $\sigma=1$. Apart from statistical precision, worse with small numbers of colloids, the concentration dependence shows to be practically negligible. It is furthermore noticeable that the values of $S_{T}$ are always positive. This agrees with the well described mass effect for Lennard-Jones mixtures, ${ }^{73-75}$ which shows that for a binary mixture of particles of different mass, the heavier particle will accumulate more effectively in the cold areas.

In order to have a reference value for the low volume fraction limit, simulations with $\phi_{c}=0.001$ have been performed with two LJ potentials. Values in Table II show, as expected, to be very similar in spite of the very different interaction among colloids. The $S_{T}$ values are positive and one order of magnitude smaller than those at higher volume fractions in Secs. V B-V D. We can conclude that in the simulations with potential interactions shown in the rest of this work, the mass effect is always present, although it is weak and does not dependent on the volume fraction.

\section{Closed packed limit}

In the limit of very high volume fraction of colloids the system will crystallize. In that case, the colloid positions are not really affected by the temperature and their density is practically constant along the temperature gradient. The separation comes then only from how the solvent still adapts to the temperature gradient. The experiments performed by Ning et $a .^{22}$ are far away from that limit. Experimental results of highly concentrated solutions of polymers, ${ }^{76,77}$ show a strong decay of the Soret coefficient to vanishing values for concentrations approaching the gelation density. In our simulations, and given the nature of the MPC solvent, the separation be- 


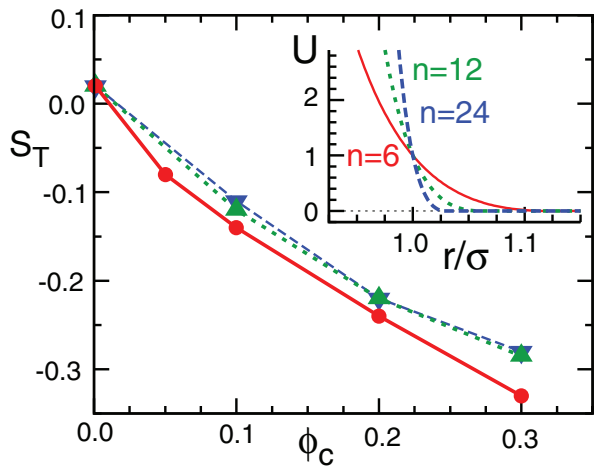

FIG. 5. Soret coefficient $S_{T}$ for different mean volume fractions $\phi_{c}$ for colloids with rLJ potentials in Eq. (10) with $n=6$ bullets, $n=12$ up-triangles, and $n=24$ down-triangles. The inset displays the employed potentials.

tween solvent and colloids remains also in the case of colloid concentration at the close packing value $S_{T, c p}$. As it will be justified in Sec. VI $S_{T, c p} \simeq-1 / T_{0}$, value that constitutes the a minimum threshold. For the simulations presented in this work then $S_{T, c p}=-0.50$.

\section{B. Effect of repulsion and repulsion softness}

With the exception of very low average volume fractions, simulations with repulsive colloid-colloid interactions show a colloid density gradient smaller than the solvent, such that the Soret coefficient is always negative, as can be seen in Fig. 5. This can be understood since the excluded volume interactions do not facilitate an inhomogeneous distribution of the colloids with the temperature gradient. Increasing concentration translates in smaller density gradients which differentiate stronger from the solvent and therefore have more negative Soret coefficients.

The repulsive interaction between colloids can range from a completely hard to very soft interactions. We investigate the effect of the repulsion softness by repulsive LennardJones type potentials in Eq. (10). An increase of the exponent $n$ is equivalent to an increase of the steepness of repulsion, or decrease of softness, as illustrated in the inset of Fig. 5. Results in Fig. 5 show a very small dependence in the repulsion softness. Although, the softer potential with $n=6$ shows magnitudes of $S_{T}$ slightly larger for all mean volume fractions than for the other two potentials, the differences are very small, and they could account for a different effective colloidal radius.

\section{Effect of attraction and attraction range}

Attractive interactions favor that colloids are on average closer to each other than colloids with purely repulsive interactions. The cold area is then more favorable since they can approach to each other decreasing their potential energy. This explains that the relative density of colloids in the cold area can eventually be higher than the solvent, which translates into a positive Soret coefficient as can be seen in Fig. 6. At low volume fractions, increasing concentration may then increase the value of $S_{T}$ from the limit of low volume fractions. Increasing the volume fraction, the repulsive part of the

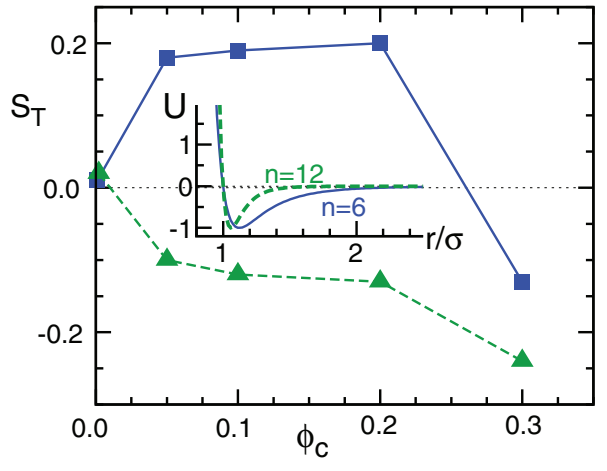

FIG. 6. Soret coefficients $S_{T}$ as a function of the volume fraction $\phi_{c}$ for LJ potentials in Eq. (10) with $n=6$ squares, $n=12$ up-triangles. The inset displays the employed potentials.

potential becomes progressively more important, and the colloids are slowly driven away from their preferred cold region. In this case the Soret coefficient decreases its value inducing eventually a sign change.

In order to investigate the effect of different attraction ranges in the thermodiffusion properties of concentrated colloidal suspensions, two types of attractive potentials are used, Lennard-Jones in Eq. (10) and sticky in Eq. (13). First, we compare two different LJ potentials as displayed in the inset of Fig. 6, the difference between these two potentials is the steepness of the repulsion (which has shown not to be of importance), and the range of the attractive interaction. Although both potentials show a decrease of $S_{T}\left(\phi_{c}\right)$ for larger volume fractions, they show a strongly different behavior. The long-ranged potential shows positive Soret coefficients for a large range of $\phi_{c}$. Meanwhile, $S_{T}$ is always negative for the shorter ranged potential. This behavior is consistent with the picture that for shorter attractions the excluded volume interaction becomes important at much smaller values of $\phi_{c}$, contributing to a considerably decrease of the $S_{T}$ values.

Figure 7 shows the importance of the attraction range also with a set of sticky potentials in Eq. (13). The functional dependence in the case of these potentials is, independently of the precise potential parameters, monotonically decaying with increasing colloidal volume fraction. In Fig. 7(a), results with three attraction ranges are presented for $\epsilon=2$. These data show a clear agreement with the results obtained with the Lennard-Jones potential, this is that decreasing the interaction range translates into smaller Soret coefficients. Figures 7(b) and 7(c) show similar results for smaller interaction strengths, $\epsilon=1$ and $\epsilon=0.5$. In this case, the Soret coefficient seems to be dominated by the repulsive interactions, not showing a significant distinction for varying interaction range.

\section{Effect of the attraction strength}

Figure 8 displays the data for the sticky potentials already shown in Fig. 7, although sorted now in different plots for fixed attraction range, in order to analyze this dependence. In all cases investigated, it is observed that an increase of the interaction strength increases the Soret coefficient. The colloids accumulate more effectively on the cold areas, where 


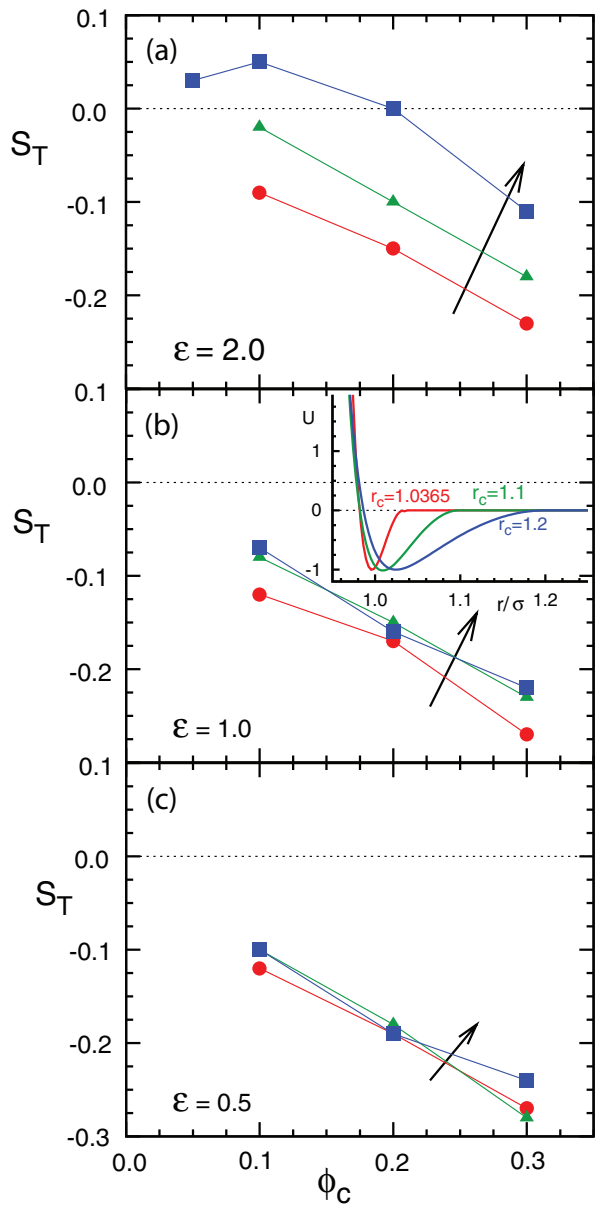

FIG. 7. Soret coefficients $S_{T}$ as a function of the volume fraction $\phi_{c}$ at various attraction ranges, squares $r_{c}=1.2$, up-triangles $r_{c}=1.1$, and bullets $r_{c}=1.0365$. Arrows indicate increasing attraction ranges. The attraction strength are (a) $\epsilon=2.0$, (b) $\epsilon=1.0$, and (c) $\epsilon=0.5$. The inset in (b) is an example of employed sticky potentials in Eq. (13).

the kinetic energy is smaller. This effect will be enhanced for colloids with stronger attraction strengths. This result is consistent with the argument discussed.

The effect of increasing attraction could be compared with the experimental data of Ning et al. ${ }^{22}$ since the employed octadecyl coated silica particles are characterized by having different attractive colloid-colloid interactions as a function of the average temperature. At low volume fractions, the trend found in our simulations agrees with the experimental trend discussed in Sec. III, namely, that increasing attraction, increases the Soret coefficient. At larger volume fractions, the experimental $S_{T}$ becomes more negative independent of the attraction, which we observe as well and interpret as the increasing importance of the repulsive interactions.

\section{ANALYTICAL APPROACH}

The definition of the Soret coefficient in stationary state in Eq. (3) can be formulated in terms the densities of each component as

$$
S_{T}=-\frac{1}{\nabla T}\left(\frac{\nabla \rho_{c}}{\rho_{c}}-\frac{\nabla \rho_{s}}{\rho_{s}}\right) .
$$

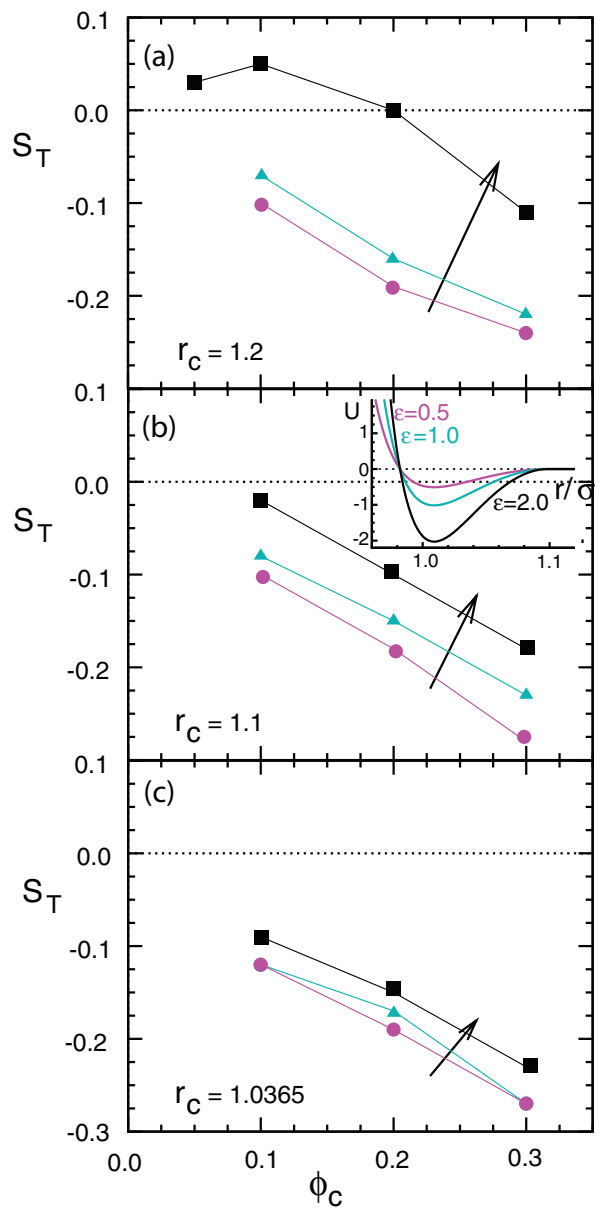

FIG. 8. Soret coefficients $S_{T}$ as a function of the volume fraction $\phi_{c}$ at various attraction ranges, squares $\epsilon=2.0$, up-triangles $\epsilon=1.0$, and bullets $\epsilon=0.5$. Arrows indicate increasing attraction strength. The attraction strength are (a) $r_{c}=1.2$, (b) $r_{c}=1.1$, and (c) $r_{c}=1.0365$. The inset in (b) is an example of employed sticky potentials in Eq. (13).

The standard definition of the thermal expansion $\beta_{T}$ can be related to the density gradient as

$$
\beta_{T}=\frac{1}{V}\left(\frac{\partial V}{\partial T}\right)_{P}=-\frac{1}{\rho}\left(\frac{\partial \rho}{\partial T}\right)_{P}=-\frac{1}{\rho}\left(\frac{\nabla \rho}{\nabla T}\right)_{P} .
$$

Equation (17) considers the density gradients of the colloids $\nabla \rho_{c}$ and of the solvent $\nabla \rho_{s}$ in the presence of both components. In a first approximation though, the contribution due to the presence of the other component can be neglected, such that each density gradient can be approximated by the equation of state of each isolated component. In this way, the Soret coefficient in Eq. (17) can be approximated by

$$
S_{T} \simeq \beta_{T, c}-\beta_{T, s} .
$$

Here $\beta_{T, s}$ is the thermal expansion coefficient of the solvent, which for the MPC fluid is known to be $\beta_{T, s}=1 / T$.

In the case of purely repulsive interactions, the thermal expansion coefficient of the colloids $\beta_{T, c}$, can be estimated by considering the Carnahan-Starling expression for hard spheres $^{78-80}$

$$
\beta_{T, c}=\frac{1}{k_{B} T}\left(\frac{1-2 \phi_{c}^{3}+\phi_{c}^{4}}{1+4 \phi_{c}+4 \phi_{c}^{2}-4 \phi_{c}^{3}+\phi_{c}^{4}}\right),
$$




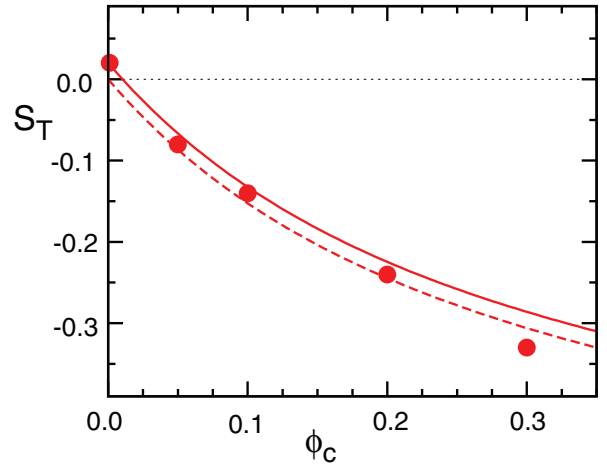

FIG. 9. Soret coefficient $S_{T}$ as a function of the volume fraction $\phi_{c}$. Symbols are simulation results with $\mathrm{rLJ} n=6$ as displayed in Fig. 5. Dashed line corresponds to Eq. (19) with $\beta_{T, c}$ in Eq. (20). Solid line includes the additional contribution $S_{T}^{m}=0.02$.

where $\phi_{c}$ is the average volume fraction of the colloids. This approximation neglects the softness of the potentials used in the simulations, which should not be a problem as discussed in Sec. V B. The mass effect discussed in Sec. V A will provide an additional contribution to the two components in Eq. (19). The value estimated in Table II as $S_{T}^{m} \simeq 0.02$ does not change the quality of the prediction shown in Fig. 9. The comparison of Eq. (19) and the simulation results in Fig. 9 shows a very good agreement. In the limit of high volume fractions discussed in Sec. V A 2, Eq. (20) is no longer valid. When the close packing volume fraction is reached, the colloid density does not vary anymore with temperature and $\beta_{T, c}=0$, which implies that $S_{T} \simeq-1 / T$.

This analysis leads to the conclusion that the collective contribution to the Soret coefficient in a colloidal suspension is mainly determined by the equations of state of each of the isolated components. In a further analysis, it will be interesting to investigate how the inclusion of the single particle effects may or not modify this conclusion. A significant effect could indicate that the interaction of each colloid with the surrounding solvent varies due to the presence of the neighbouring colloids.

\section{DISCUSSION}

Two additional aspects of the comparison of our simulations with existing investigations are important to be emphasized. On the one hand, the theoretical work of Dhont ${ }^{20,21}$ considers an incompressible fluid, and although the experimental system ${ }^{22}$ is not perfectly incompressible, it will definitely have a different equation of state than the ideal gas behavior of the MPC fluid. The qualitative agreement of the results validates the model, although this limitation should be taken into account.

A second aspect to be considered is that in the experimental work, ${ }^{22}$ different temperatures are related with different colloid-colloid interactions. Meanwhile, the theoretical approach in Eq. (6) is performed for hard-sphere colloids, assuming that the single and collective contributions for the thermal diffusion coefficient are independent of each other, as discussed in Sec. II. This means that the dependence of the Soret coefficient on volume fraction at low concentration in Eq. (6) only relies on the different single particle contributions. Our simulations clearly take different colloid-colloid interactions into account with a unique single particle contribution. The trend displayed is in principle similar to the one in experiments, although the experimental dependence in the low volume fraction regime should be carefully taken into account given the large experimental errors in the considered relative quantities. The agreement in the trend of our simulations and of the experiments arising from the different colloidcolloid interactions would support that the single and collective contributions are independent for the Soret coefficient but not for the self-diffusion coefficient. This conclusion should be though verified in the case of a more accurate model, where the single particle contribution to the thermodiffusion coefficient is more explicitly taken into account.

\section{SUMMARY AND CONCLUSIONS}

In this paper, a simulation study of the thermodiffusion properties of concentrated colloidal suspensions is presented. Our model considers the presence of colloids with explicit solvent particles and disregards the specific interactions of the colloids surface with the surrounding solvent. These interactions are responsible for the single particle effects which are the main contribution to the Soret coefficient. This simulation model, therefore, allows us to separately investigate the effect of the much less understood contribution of collective interactions to the Soret coefficient.

The experimental data of Ning et al. ${ }^{22}$ are first discussed and reanalyzed by subtracting the single particle contribution. This enables us to perform the comparison of our simulation data with experiments. Our simulation study considers different colloid-colloid interactions. These are purely repulsive, or repulsive combined with attraction. For the repulsive potentials, we consider several softness at short distances, while with the attraction we study the effect of the range and strength of the attraction with two different potential shapes. The main trends found in our study can be summarized as follows. Repulsive interactions among colloids disfavor an inhomogeneous distribution of the colloids with the temperature gradient. In our simulation model, this translates into negative values of the Soret coefficient of increasing magnitude with increasing concentration. In contrast, attractive interactions favor an inhomogeneous distribution of the colloids with the temperature gradient, which translates into a more effective accumulation of the colloids in the cold areas and, therefore, higher values of the Soret coefficient. In this way, increasing the concentration, always increases the importance of the repulsive interaction, such that the $S_{T}$ values decrease. On the other hand, increasing the range or strength of the attraction leads to larger values of $S_{T}$, while changing details in the interaction like potential shape or repulsion steepness is relatively unimportant. These trends nicely explain all our simulation results qualitatively. Experimental results also follow this behavior, namely, that $S_{T}$ increases with increasing attraction at low volume fractions and $S_{T}$ decreases with increasing concentration at high volume fraction. Furthermore, a reasonable quantitative agreement between simulation and experimental results is found, as discussed in Sec. IV E. 
A theoretical estimation of $S_{T}$ for colloidal suspensions with repulsive colloid-colloid interactions is obtained by assuming that colloids and fluid distribute along the temperature gradient following their individual equations of state, this is disregarding the mutual interactions. Although complementary studies are still desirable, the good agreement between this estimation and our simulation result indicates that the collective contribution to the Soret coefficient in concentrated colloidal suspensions can be determined from the equilibrium properties of each of the system components. Additional experimental results and further simulations of concentrated colloidal systems taking into account more explicit colloid-solvent interactions will bring valuable information. These results could support the approach made in our simulation model about the independence of the single particle and collective contributions to the Soret coefficient. This would be on the one hand an important concept in the fundamental understanding of the thermodiffusion effect. On the other hand, it could help in the development of practical applications of thermodiffusion in concentrated systems, such as thermal flow field fractionation.

\section{ACKNOWLEDGMENTS}

The authors want to thank helpful discussions with Simone Wiegand, Gerrit A. Vliegenthart, Mingcheng Yang, and Gerhard Gompper. We also want to acknowledge S. Wiegand for making directly available to us experimental data in Ref. 22 and to G. A. Vliegenthart for proofreading the paper.

${ }^{1}$ S. Wiegand, J. Phys.: Condens. Matter 16, R357 (2004).

2 J. Giddings, Science 260, 1456 (1993).

${ }^{3}$ D. Vigolo, R. Rusconi, H. A. Stone, and R. Piazza, Soft Matter 6, 3489 (2010).

${ }^{4}$ S. Duhr and D. Braun, Proc. Natl. Acad. Sci. U.S.A. 103, 19678 (2006).

${ }^{5}$ H. R. Jiang, H. Wada, N. Yoshinaga, and M. Sano, Phys. Rev. Lett. 102, 208301 (2009).

${ }^{6}$ H. R. Jiang, N. Yoshinaga, and M. Sano, Phys. Rev. Lett. 105, 268302 (2010).

${ }^{7}$ P. Baaske, F. M. Weinert, S. Duhr, K. H. Lemke, M. J. Russell, and D. Braun, Proc. Natl. Acad. Sci. U.S.A. 104, 9346 (2007).

${ }^{8}$ I. Budin, R. J. Bruckner, and J. W. Szostak, J. Am. Chem. Soc. 131, 9628 (2009).

${ }^{9}$ E. Bringuier and A. Bourdon, Phys. Rev. E 67, 011404 (2003).

${ }^{10}$ E. Bringuier and A. Bourdon, Physica A 385, 9 (2007).

${ }^{11}$ A. Würger, Phys. Rev. Lett. 98, 138301 (2007).

${ }^{12}$ R. Piazza and A. Parola, J. Phys.: Condens. Matter 20, 153102 (2008).

${ }^{13}$ A. Würger, Rep. Prog. Phys. 73, 126601 (2010).

${ }^{14}$ D. Rings, R. Schachoff, M. Selmke, F. Cichos, and K. Kroy, Phys. Rev. Lett. 105, 090604 (2010).

${ }^{15}$ S. Wongsuwarn, D. Vigolo, R. Cerbino, A. M. Howe, A. Vailati, R. Piazza, and P. Cicuta, Soft Matter 8, 5857 (2012).

${ }^{16}$ R. Piazza and A. Guarino, Phys. Rev. Lett. 88, 208302 (2002).

${ }^{17}$ J. Rauch and W. Köhler, Phys. Rev. Lett. 88, 185901 (2002).

${ }^{18}$ N. Ghofraniha, G. Ruocco, and C. Conti, Langmuir 25, 12495 (2009).

${ }^{19}$ A. Majee and A. Würger, Phys. Rev. E 83, 061403 (2011).

${ }^{20}$ J. K. G. Dhont, J. Chem. Phys. 120, 1632 (2004).

${ }^{21}$ J. K. G. Dhont, J. Chem. Phys. 120, 1642 (2004).

${ }^{22}$ H. Ning, J. Buitenhuis, J. K. G. Dhont, and S. Wiegand, J. Chem. Phys. 125, 204911 (2006).

${ }^{23}$ S. R. de Groot and P. Mazur, Nonequilibrium Thermodynamics (Dover, New York, 1984).

${ }^{24}$ M. Yang and M. Ripoll, J. Phys.: Condens. Matter 24, 195101 (2012).

${ }^{25}$ S. Duhr and D. Braun, Phys. Rev. Lett. 96, 168301 (2006).

${ }^{26}$ S. A. Putnam, D. G. Cahill, and G. C. L. Wong, Langmuir 23, 9228 (2007).

${ }^{27}$ M. Braibanti, D. Vigolo, and R. Piazza, Phys. Rev. Lett. 100, 108303 (2008).
${ }^{28}$ H. Brenner, Phys. Rev. E 74, 036306 (2006).

${ }^{29}$ D. H. Napper, J. Colloid Interface Sci. 58, 390 (1980).

${ }^{30}$ G. A. Vliegenthart, J. S. van Duijneveldt, and B. Vincent, Faraday Discuss. 123, 65 (2003).

${ }^{31}$ In Ref. 22, this behavior has been by mistake described as a power law dependence.

${ }^{32} \mathrm{M}$. Vladkov and J. L. Barrat, Nano Lett. 6, 1224 (2006).

${ }^{33}$ G. Galliéro and S. Volz, J. Chem. Phys. 128, 064505 (2008).

${ }^{34}$ L. Joly, S. Merabia, and J. L. Barrat, EPL 94, 50007 (2011).

${ }^{35}$ G. R. McNamara and G. Zanetti, Phys. Rev. Lett. 61, 2332 (1988).

${ }^{36}$ P. J. Hoogerbrugge and J. M. V. A. Koelman, EPL 19, 155 (1992).

${ }^{37}$ F. J. Alexander, S. Chen, and J. D. Sterling, Phys. Rev. E 47, 2249(R) (1993).

${ }^{38}$ P. Lallemand and L.-S. Luo, Phys. Rev. E 68, 036706 (2003).

${ }^{39}$ P. Español, EPL 40, 631 (1997).

${ }^{40}$ J. Bonet-Avalós and A. D. Mackie, EPL 40, 141 (1997).

${ }^{41}$ M. Ripoll and M. H. Ernst, Phys. Rev. E 71, 041104 (2005).

${ }^{42}$ A. Malevanets and R. Kapral, J. Chem. Phys. 110, 8605 (1999).

${ }^{43}$ T. Ihle and D. M. Kroll, Phys. Rev. E 63, 020201(R) (2001).

${ }^{44}$ J. T. Padding and A. A. Louis, Phys. Rev. E 74, 031402 (2006).

${ }^{45}$ A. Malevanets and R. Kapral, J. Chem. Phys. 112, 7260 (2000).

${ }^{46}$ M. Ripoll, K. Mussawisade, R. G. Winkler, and G. Gompper, EPL 68, 106 (2004).

${ }^{47}$ D. Lüsebrink and M. Ripoll, J. Chem. Phys. 136, 084106 (2012).

${ }^{48}$ E. Tüzel, M. Strauss, T. Ihle, and D. M. Kroll, Phys. Rev. E 68, 036701 (2003).

${ }^{49}$ N. Kikuchi, C. M. Pooley, J. F. Ryder, and J. M. Yeomans, J. Chem. Phys. 119, 6388 (2003).

${ }^{50}$ E. Tüzel, T. Ihle, and D. M. Kroll, Phys. Rev. E 74, 056702 (2006).

${ }^{51}$ M. Ripoll, K. Mussawisade, R. G. Winkler, and G. Gompper, Phys. Rev. E 72, 016701 (2005).

${ }^{52}$ D. Lüsebrink, M. Yang, and M. Ripoll, J. Phys.: Condens. Matter 24, 284132 (2012).

${ }^{53}$ M. Yang and M. Ripoll, Phys. Rev. E 84, 061401 (2011).

${ }^{54}$ R. G. Winkler, M. Ripoll, K. Mussawisade, and G. Gompper, Comput. Phys. Commun. 169, 326 (2005).

${ }^{55}$ K. Mussawisade, M. Ripoll, R. G. Winkler, and G. Gompper, J. Chem. Phys. 123, 144905 (2005).

${ }^{56}$ R. G. Winkler, K. Mussawisade, M. Ripoll, and G. Gompper, J. Phys.: Condens. Matter 16, S3941 (2004).

${ }^{57}$ M. Ripoll, P. Holmqvist, R. G. Winkler, G. Gompper, J. K. G. Dhont, and M. P. Lettinga, Phys. Rev. Lett. 101, 168302 (2008).

${ }^{58}$ M. Ripoll, R. G. Winkler, K. Mussawisade, and G. Gompper, J. Phys.: Condens. Matter 20, 404209 (2008).

${ }^{59}$ M. P. Lettinga, J. K. G. Dhont, Z. Zhang, S. Messlinger, and G. Gompper, Soft Matter 6, 4556 (2010).

${ }^{60}$ M. Ripoll, R. G. Winkler, and G. Gompper, Phys. Rev. Lett. 96, 188302 (2006).

${ }^{61}$ M. Ripoll, R. G. Winkler, and G. Gompper, Eur. Phys. J. E 23, 249 (2007).

${ }^{62}$ S. P. Singh, R. G. Winkler, and G. Gompper, Phys. Rev. Lett. 107, 158301 (2011).

${ }^{63}$ J. Elgeti and G. Gompper, EPL 85, 38002 (2009).

${ }^{64}$ J. Elgeti, U. B. Kaupp, and G. Gompper, Biophys. J. 99, 1018 (2010).

${ }^{65}$ S. Y. Reigh, R. G. Winkler, and G. Gompper, Soft Matter 8, 4363 (2012).

${ }^{66}$ G. A. Vliegenthart, J. F. M. Lodge, and H. N. W. Lekkerkerker, Physica A 263, 378 (1999).

${ }^{67}$ S. Asakura and F. Oosawa, J. Chem. Phys. 22, 1255 (1954).

${ }^{68}$ A. Vrij, Pure Appl. Chem. 48, 471 (1976).

${ }^{69}$ P. Melby, A. Prevost, D. A. Egolf, and J. S. Urbach, Phys. Rev. E 76, 051307 (2007).

${ }^{70}$ J. A. Barker and D. Henderson, Rev. Mod. Phys. 48, 587 (1976).

${ }^{71}$ B. Hafskjold, T. Ikeshoji, and S. K. Ratkje, Mol. Phys. 80, 1389 (1993).

${ }^{72}$ F. Müller-Plathe, J. Chem. Phys. 106, 6082 (1997).

${ }^{73}$ D. Reith and F. Müller-Plathe, J. Chem. Phys. 112, 2436 (2000).

${ }^{74}$ G. Galliéro, B. Duguay, J.-P. Caltagirone, and F. Montel, Fluid Phase Equilib. 208, 171 (2003).

${ }^{75}$ G. Galliéro, B. Duguay, J.-P. Caltagirone, and F. Montel, Philos. Mag. 83, 2097 (2003).

${ }^{76}$ J. Rauch and W. Köhler, Macromolecules 38, 3571 (2005).

${ }^{77}$ J. Rauch, M. Hartung, A. F. Privalov, and W. Köhler, J. Chem. Phys. 126, 214901 (2007).

${ }^{78}$ N. F. Carnahan and K. E. Starling, J. Chem. Phys. 51, 635 (1969).

${ }^{79}$ N. F. Carnahan and K. E. Starling, J. Chem. Phys. 53, 600 (1970).

${ }^{80}$ E. Wilhelm, Monatsch. Chem. 105, 291 (1974). 